\title{
Macular perfusion analysed by optical coherence tomography angiography after uncomplicated phacoemulsification: benefits beyond restoring vision
}

Ana Križanović ${ }^{1,2}$, Mirjana Bjeloš ${ }^{1,2,3^{*}}$, Mladen Bušić ${ }^{1,2,3}$, Biljana Kuzmanović Elabjer ${ }^{1,2,3}$, Benedict Rak and Nenad Vukojević $c^{4,5}$

\begin{abstract}
Background: The purpose of the study is to investigate the changes of macular perfusion by OCT-angiography (OCT-A) after uncomplicated phacoemulsification.

Methods: OCT-A was performed before cataract surgery, 1 week, 1 month, and 3 months after surgery recording superficial vascular complex (SVC), nerve fiber layer vascular plexus (NFLVP), superficial vascular plexus (SVP), deep vascular complex (DVC), intermediate capillary plexus (ICP) and deep capillary plexus (DCP), as well as large choroidal blood vessels and choriocapillaris (CC). Explant area (EA), vessels area (VA), vessels percentage area (VPA), total number of junctions (TNJ), junctions density (JD), total vessels length (TVL), average vessels length (AVL), total number of end points (TNEP), and mean lacunarity (ML) throughout all layers were analysed.

Results: Significant changes of vascular parameters in 55 eyes of 55 patients mostly reached plateau one week after surgery and remained stable up to $3 \mathrm{~m}$ after surgery, occurring in all retinal layers but not in choroid and CC. The greatest increase in VPA (22.79\%), TVL (16.71\%), AVL (166.71\%) and JD (29.49\%) was in SVC. On the contrary, the greatest change of ML (-53.41\%) appeared in DVC.

Conclusions: This is the first OCT-A study demonstrating perfusion alterations in macula after phacoemulsification due to functional hyperaemia. We presume the effect is evoked by increased light intensity stimulation of retina after cataract removal. Accordingly, phacoemulsification in elderly population could have advantageous feature in addition to restoring visual acuity.
\end{abstract}

Keywords: Macula, Angiography, Retinal vessels, Blood supply, Phacoemulsification, Cataract

\footnotetext{
* Correspondence: dr.mbjelos@gmail.com

${ }^{1}$ Department of Ophthalmology, Reference Centre of the Ministry of Health

of the Republic of Croatia for Paediatric Ophthalmology and Strabismus,

University Hospital "Sveti Duh", Sveti Duh 64, Zagreb, Croatia

${ }^{2}$ Faculty of Dental Medicine and Health Osijek, Josip Juraj Strossmayer

University of Osijek, Osijek, Croatia

Full list of author information is available at the end of the article
}

(c) The Author(s). 2021 Open Access This article is licensed under a Creative Commons Attribution 4.0 International License, which permits use, sharing, adaptation, distribution and reproduction in any medium or format, as long as you give appropriate credit to the original author(s) and the source, provide a link to the Creative Commons licence, and indicate if changes were made. The images or other third party material in this article are included in the article's Creative Commons licence, unless indicated otherwise in a credit line to the material. If material is not included in the article's Creative Commons licence and your intended use is not permitted by statutory regulation or exceeds the permitted use, you will need to obtain permission directly from the copyright holder. To view a copy of this licence, visit http://creativecommons.org/licenses/by/4.0/ The Creative Commons Public Domain Dedication waiver (http://creativecommons.org/publicdomain/zero/1.0/) applies to the data made available in this article, unless otherwise stated in a credit line to the data. 


\section{Background}

The exact impact of phacoemulsification, one of the most common surgical procedures in the world [1], on macular perfusion is unknown [2, 3]. Changes in arterial blood pressure, position of blood vessels, venous return of blood and $\mathrm{CO}_{2}$ levels all influence eye perfusion during cataract surgery [4]. Within retina, one of the tissues with the highest metabolic requirements in the body $[5,6]$, three layers of the retinal blood vessels exist: superficial, deep, and intermediate layer [6]. Superficial vascular complex (SVC), formed of nerve fiber layer vascular plexus (NFLVP) and superficial vascular plexus (SVP), is located in the nerve fiber layer (NFL) and ganglion cell layer (GCL) respectively [5]. Deep vascular complex (DVC), formed of intermediate capillary plexus (ICP) and deep capillary plexus (DCP), is located deeper in the inner nuclear (INL) and outer plexiform layer (OPL) [5]. Inner plexiform layer (IPL) is thus supported by two capillary networks: SVP and ICP. The superficial layer contains arterioles, venules, and capillaries, while the deep layer consists of capillary-sized blood vessels [5].

The aim of this study was to investigate the changes of macular perfusion analysed by optical coherence tomography angiography (OCT-A) after uncomplicated phacoemulsification in normal ageing subjects. If such changes do exist, it is necessary to define whether they are favorable or adverse and thus to provide an evidence based recommendation for the indication of cataract surgery if patients can reach further benefits beyond improvement in visual acuity.

\section{Methods}

\section{Patients}

The aim of the study was to investigate the changes of macular perfusion by OCT-A after uncomplicated phacoemulsification. The study was conducted at the Department of Ophthalmology, University Hospital "Sveti Duh" Zagreb on one group of patients scheduled to have microincision phacoemulsification under topical anesthesia. All patients were operated by the same surgeon (BKE) and monitored and analysed by one examiner (AK). The analysed parameters refer to only one eye.

The inclusion criteria for the study were: 1 . uncomplicated senile cataract; 2 . cataract Pentacam ${ }^{\circ}$ Nucleus Staging (PNS) assessed as 1, 2 or 3 by Oculus Pentacam ${ }^{\circ}$ nucleus grading system; 3. Quality index (Q) of OCT-A images $\geq 30$; 4 . axial length (AL) $20-25 \mathrm{~mm}$ measured by optical biometry; 5 . intraocular pressure (IOP) measured by Goldmann tonometry $10-21 \mathrm{mmHg} ; 6$. systolic blood pressure $(\mathrm{SBP}) \geq 90$ and $\leq 140 \mathrm{mmHg}$, and diastolic blood pressure $(\mathrm{DBP}) \geq 60$ and $\leq 90 \mathrm{mmHg}$.
The exclusion criteria for the study were: corneal diseases, pseudoexfoliation syndrome, cataracts other than uncomplicated senile, glaucoma, age-related macular degeneration, signs of hypertensive retinopathy [7], degenerative myopia, diabetes, intraoperative and/or postoperative complications, and poor image quality.

\section{Methods}

All patients underwent history taking, determination of best corrected visual acuity (BCVA), auto-refractokeratometry, endothelial biomicroscopy, optical biometry, arterial pressure measurement, IOP measurement by Goldmann tonometry, optical coherence tomography (OCT) of the macula, fundus examination in mydriasis and OCT-A imaging.

BCVA was determined using the ETDRS table and recorded in the $\log$ MAR unit [8]. Corneal endothelial cells were analysed by specular biomicroscope (CEM-530, Nidek, Gamagori, Japan). The AL was determined using optical biometer (IOLMaster $700^{\circ}$, Zeiss, Oberkochen, Germany). Arterial pressure was measured with a digital pressure gauge (M6 Comfort, Omron, Kyoto, Japan) and IOP by Goldmann applanation tonometry. Mean arterial pressure (MAP) and ocular perfusion pressure (OPP) were calculated using $\mathrm{MAP}=(\mathrm{SBP}+2 \mathrm{x} \mathrm{DBP}) / 3$ and $\mathrm{OPP}=2 \mathrm{x}$ (MAP - IOP) / 3 formulas respectively. Examination of the anterior segment of the eye was performed using a slit lamp. Tropicamide 1\% (Mydriacyl ${ }^{\circ}$, Alcon Laboratories Inc., Geneva, Switzerland) was dripped into each eye of the patient three times at intervals of $15 \mathrm{~min}$ to maximize mydriasis and cycloplegia. OCT (FAST mode recording $20 \times 20^{\circ}$ of the central macula using 512 A-scans $\times 25$ sections with $240 \mu \mathrm{m}$ distance between sections) and autofluorescence (multi-color, infrared, blue and green) were recorded using HRA + OCT Spectralis ${ }^{\circ}$ (Heidelberg Engineering, Heidelberg, Germany). Each patient underwent objective refraction measuring using auto-refractokeratometer (Righton Speedy-K Autorefractor Keratometer, Right Group, Tokyo, Japan), imaging on Pentacam ${ }^{\bullet}$ HR device (OCULUS Optikgeräte GmbH, Wetzlar, Germany) to calculate PNS and fundus examination with an indirect ophthalmoscope.

OCT-A was performed on HRA + OCT Spectralis device recording $10 \times 10^{\circ}$ of the central macula, accordingly $2.9 \times 2.9 \mathrm{~mm}$, with 512 A-scans $\times 512$ sections, $6 \mu \mathrm{m}$ distance between sections and resolution of $5.7 \mu \mathrm{m} /$ pixel. Automated segmentation performed with integrated Spectralis software of superficial layer (NFLVP and SVP forming SVC) and deep layer (DCP and ICP forming DVC) of retinal vessels, CC and large choroidal blood vessels is considered reliable in case of unremarkable retinal layer pathology [9]. Recording was done using high resolution mode, reported for low measurement variability $[10,11]$. In case of possible misleading artefacts, the measurement was repeated. Images 
with quality index $Q \geq 30$, as computed by integrated software, were further analysed and carefully reviewed by two graders for accurate segmentation of the vascular layers and presence of artefacts prior to final analysis [9].

Patients were evaluated before and 7 days, 1 month, and 3 months after surgery. Images were exported to AngioTool 0.6 software for quantitative analysis [12]. A vessel is defined after segmentation as a segment between two branching points or a branching point and an end point. After segmentation, vessels were skeletonized and analysed (Fig. 1) [12]. OCT-A vascular parameters included: explant area (EA): the analysed area; vessels area (VA): area of the segmented vessels; vessels percentage area (VPA): percentage of area containing vessels inside the explant area (VA/ $\mathrm{EA}$ ); total number of junctions (TNJ): total number of vessels junctions in the image; junctions' density (JD): number of vessel junctions per unit area (branch points/unit area); total vessels length (TVL): sum of Euclidean distances between the pixels of all the vessels in the image; average vessels length (AVL): mean length of all the vessels in the image; total number of end points (TNEP): number of open-ended segments; mean lacunarity (ML): mean lacunarity over all size boxes.

Foldable intraocular lens (Zeiss CT Lucia 611PY or AMO Tecnis PCB00) were implanted. Total cumulative dissipated energy (CDE) and total ultrasound time (PHACO time) were automatically recorded using the Centurion Vision System (Alcon Inc., Fort Worth, USA) [13]. Postoperatively dexamethasone $0.1 \%$ drops (Maxidex ${ }^{\oplus}$, Alcon Laboratories Inc., Geneva, Switzerland) were prescribed q.i.d. for 7 days followed by b.i.d. for 7 days.

\section{Statistical analysis}

Statistical analysis was performed using MedCalc statistical software (MedCalc Software Ltd., Ostend, Belgium). Data are presented by median and interquartile ranges. A comparison of pre-operative values with values obtained one week, one month, and three months after surgery was made using non-parametric Friedman

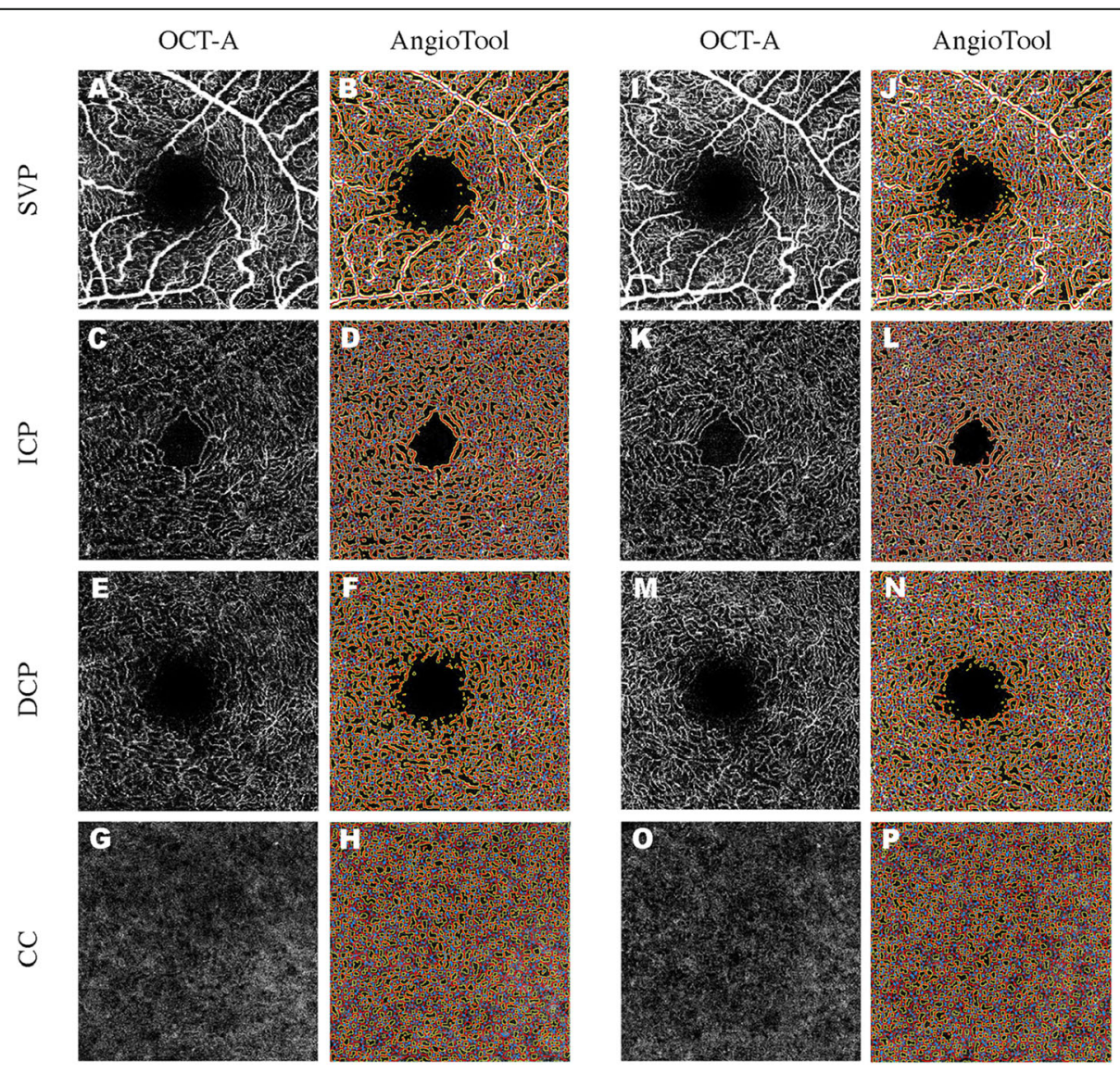

Fig. 1 OCT-A en-face images (image quality Q =35) showing binary skeletal structure in the left column $\mathbf{a}, \mathbf{c}, \mathbf{e}, \mathbf{g}, \mathbf{i}, \mathbf{k}, \mathbf{m}, \mathbf{o}$ and skeletonized images with overlay of the AngioTool output in the corresponding right column $\mathbf{b}, \mathbf{d}, \mathbf{f}, \mathbf{h}, \mathbf{j}, \mathbf{l}, \mathbf{n}, \mathbf{p}$ before and 1 week after surgery. The skeleton is outlined in red, vessels are presented in yellow, branching points are indicated in blue. Calculated vascular parameters are presented in the Additional file 1: Table S11 
ANOVA test. Inter-layer comparison of vascular parameters three months after surgery was made using Student's $t$ test. The significance level was set to $P<0.05$.

\section{Results}

Fifty-nine patients met the inclusion criteria, yet 55 eyes of 55 patients were included in final analysis. One patient developed postoperative uveitis and was excluded from further analysis. Two patients missed the follow-up appointments. One patient developed pseudophakic macular edema (PME) $1 \mathrm{~m}$ after surgery based on OCT criteria [14], with no signs of PME three months after surgery. General characteristics of the participants, and surgery parameters are presented in the Additional file 1: Table S1.

\section{General parameters}

IOP before surgery was significantly higher than one week, one month and three months after surgery. IOP one week after surgery was significantly higher than one month and three months after surgery. SBP before surgery was significantly higher than one month and three months after surgery. BCVA significantly improved one week after surgery, with further significant improvement when comparing one week and three months after surgery. DBP, MAP and OPP did not display any change (Additional file 1: Table S2).

\section{Quality index}

Average quality of OCT-A images before and after phacoemulsification demonstrated no significant changes (Table 1).

\section{Vascular parameters}

No difference was found in EA. An increase in parameters: VA, VPA, TNJ, JD, TVL and AVL was found in all layers except in CC and choroid. A decrease in parameter TNEP was found in all layers except in choroid and NFLVP. ML decreased in all layers but choroid.

The most pronounced changes were found in NFLVP and SVC. Average vessels length has undergone the greatest change in all layers except in NFLVP. For most vascular parameters a statistically significant difference was observed between values obtained before surgery and values obtained one week, one month, and three months after surgery within which there was no statistically significant difference (Additional file 1 : Table S3; Table S4).

Changes of vascular parameters in SVP, ICP, DCP and $\mathrm{CC}$ are presented in Fig. 1.

\section{Nerve fiber layer vascular plexus, superficial vascular plexus and superficial vascular complex}

In NFLVP layer, VA, VPA, TNJ, JD, TVL and AVL one week after surgery were lower than one month and three months after surgery, within which there was no significant difference (Table 2). For TNEP no alterations were detected (Table 2).

In SVP and SVC an increase in VA, VPA, TNJ, JD and TVL with decrease in TNEP and ML was found one week postoperatively (Table 3; Table 4). In addition, TNEP was higher one week after surgery than three months after surgery (Table 3; Table 4). In SVP layer, TVL one week after surgery was lower than three months after surgery (Table 3). In SVC layer TNJ, JD, TVL and AVL values were lower one week compared to three months after surgery (Table 4).

\section{Intermediate capillary plexus, deep capillary plexus and deep vascular complex}

In ICP and DVC an increase in VA, VPA, TNJ, JD, TVL and AVL along with decrease in TNEP and ML was found one week postoperatively. No difference between one week, one month and three months after surgery was observed for any parameter. In addition, the largest significant change was observed for AVL (75.78 and $113.40 \%$ respectively) (Table 5; Table 6).

In DCP layer an increase in TNJ, JD, TVL and AVL and decrease in TNEP and ML was found postoperatively one week, one month and three months after surgery with no significant difference between them. VA and VPA before surgery were lower than one week, one month and three months after surgery. VA and VPA one week after surgery were lower than three months after surgery. In DCP, the largest statistically significant change in preoperative versus postoperative value was observed for AVL (103.51\%) (Table 7).

\section{Retina}

Observing the whole retina, an increase in VA, VPA, TNJ, JD, TVL and AVL with decrease in TNEP and ML was found one week postoperatively. No difference

Table 1 Quality index of OCT-A images before and after phacoemulsification

\begin{tabular}{llllll}
\hline OCT-A image & Before & 1 week after & 1 month after & 3 months after & $\boldsymbol{P}$ \\
\hline Quality index & $34(31-37.5)$ & $34(32-36.8)$ & $35(32.25-37.75)$ & $35(33-38)$ & 0.170 \\
\hline$O C T-A ~$
\end{tabular}

OCT-A optical coherence tomography angiography, $Q$ quality index

This table shows median and interquartile ranges for quality index (25th and 75th percentiles)

Friedman ANOVA test, the significance level was set to $P<0.05$ 
Table 2 Statistical analysis of changes in vascular parameters in nerve fiber layer vascular plexus

\begin{tabular}{|c|c|c|c|c|c|c|}
\hline NFLVP & Before & 1 week after & 1 month after & 3 months after & $P$ & Bias \\
\hline$\overline{E A}\left(m^{2}\right)$ & $8.3791(8.3755-8.3804)$ & 8.3796 (8.3770-8.3805) & $8.3794(8.3768-8.3807)$ & 8.3794 (8.3759-8.3803) & 0.740 & $0.05 \%$ \\
\hline$V A\left(m^{2}\right)$ & $1.7746(1.3408-2.4250)$ & $2.3389(1.7994-2.7072)$ & $2.4731(2.0283-3.0603)$ & $2.4584(1.9830-2.9862)$ & $<0.001^{*}$ & $31.72 \%^{*}$ \\
\hline VPA (\%) & $21.1911(16.0203-28.9405)$ & $27.9786(21.4717-32.3195)$ & $29.5066(24.2454-36.5190)$ & $29.3376(23.6625-35.6347)$ & $<0.001^{*}$ & $31.67 \%^{*}$ \\
\hline TNJ & $456(300-725)$ & $692(457-871)$ & $781(583-1000)$ & $729(558-996)$ & $<0.001^{*}$ & $44.48 \%^{*}$ \\
\hline JD (junctions $/ \mathrm{mm}^{2}$ ) & $54.5141(35.8141-86.5340)$ & $82.5760(54.5604-103.8807)$ & $93.3164(69.6196-119.4013)$ & 86.9921 (66.5249-118.8970) & $<0.001^{*}$ & $44.43 \%{ }^{*}$ \\
\hline TVL (mm) & 66.7446 (51.4339-93.6245) & $87.9231(70.0598-102.8570)$ & 94.7294 (76.9631-114.5119) & $92.3583(78.0480-112.1177)$ & $<0.001^{*}$ & $29.22 \%^{*}$ \\
\hline AVL (mm) & $0.1761(0.1287-0.2544)$ & $0.2275(0.1656-0.3089)$ & $0.2604(0.1823-0.3953)$ & $0.2691(0.1864-0.3845)$ & $<0.001^{*}$ & $24.72 \%^{*}$ \\
\hline TNEP & 954 (864-1031) & $984(874-1061)$ & 966 (855-1059) & 959 (850-1124) & 0.426 & $4.39 \%$ \\
\hline ML & $0.2865(0.2111-0.4280)$ & $0.2288(0.1935-0.3086)$ & $0.2311(0.1543-0.2945)$ & $0.2151(0.1603-0.2506)$ & $<0.001$ & $-31.15 \%$ \\
\hline
\end{tabular}

NFLVP nerve fiber layer vascular plexus, EA explant area, VA vessels area, VPA vessels percentage area, TNJ total number of junctions, JD junctions density, $T V L$ total vessels length, $A V L$ average vessels length, TNEP total number of end points, $M L$ mean lacunarity

This table shows median and interquartile ranges for each parameter (25th and 75th percentile). $P$ values and percentages of change are presented one week after phacoemulsification

Friedman ANOVA test, significant difference (bold values) was found for values with $P<0.05$

*Observed values were lower one week compared to one and three months after surgery, within which there was no significant difference

between one week, one month and three months after surgery was observed for any parameter (Table 8).

\section{Choriocapillaris}

In choriocapillaris TNEP was higher before surgery than one week, one month and three months after surgery, with no significant difference between them. ML before surgery was higher than one month and three months after surgery (Additional file 1: Table S5).

\section{Choroid}

No statistically significant changes in vascular parameters were observed (Additional file 1: Table S6).

\section{Inter-layer differences}

Morphometric differences between the vascular layers are presented online in Additional file 1: Tables S7-S10.

\section{Discussion}

This study monitored vascular parameters of retinal blood vessels across NFLVP, SVP, SVC, ICP, DCP, DVC, as well as $\mathrm{CC}$ and large and medium choroidal blood vessels revealing that uncomplicated phacoemulsification significantly improved macular hemodynamics. We consider these changes to be favorable and beneficial.

\section{Major results}

A significant increase in VA, VPA, TNJ, JD, TVL and AVL was found, followed by the decrease in TNEP and ML manifesting rise in blood supply of the central macula after phacoemulsification (Additional file 1: Table S3; Table S4). Most changes in vascular parameters were evident one week after surgery and remained stable up to three months after surgery. These changes affected all retinal layers but not $\mathrm{CC}$ and choroid (Additional file 1: Table S5; Table S6). Observed patterns of alterations between the retina and choroid were somewhat expected

Table 3 Statistical analysis of changes in vascular parameters in superficial vascular plexus

\begin{tabular}{|c|c|c|c|c|c|c|}
\hline SVP & Before & 1 week after & 1 month after & 3 months after & $P$ & Bias \\
\hline $\mathrm{EA}\left(\mathrm{mm}^{2}\right)$ & 8.3792 (8.3777-8.3806) & 8.3794 (8.3780-8.3806) & 8.3801 (8.3793-8.3807) & 8.3801 (8.3790-8.3809) & 0.066 & $0.01 \%$ \\
\hline$V A\left(\mathrm{~mm}^{2}\right)$ & 4.4132 (3.8255-4.9965) & $4.9826(4.6831-5.2155)$ & $5.0491(4.7088-5.3848)$ & $5.0094(4.7490-5.4082)$ & $<0.001$ & $15.00 \%$ \\
\hline VPA (\%) & $52.6630(45.6480-59.7126)$ & $59.4532(55.8881-62.2341)$ & $60.2560(56.1915-64.2580)$ & $59.7772(56.6644-64.6154)$ & $<0.001$ & $14.93 \%$ \\
\hline TNJ & $1242(1086-1377)$ & $1398(1337-1467)$ & $1421(1345-1495)$ & $1433(1364-1507)$ & $<0.001$ & $16.58 \%$ \\
\hline JD (junctions/mm²) & $148.2088(129.6466-164.2696)$ & $166.8051(159.6096-175.0851)$ & 169.5579 (160.4704-178.4466) & $170.9742(162.8021-179.8361)$ & $<0.001$ & $16.50 \%$ \\
\hline TVL (mm) & 135.6119 (126.7246-143.7911) & 145.2219 (140.3096-149.2926) & $147.1460(142.4993-150.2033)$ & $146.6076(141.8954-151.0425)$ & $<0.001^{*}$ & $9.56 \%^{*}$ \\
\hline $\mathrm{AVL}(\mathrm{mm})$ & 3.3768 (1.5705-5.5304) & 6.7711 (4.8339-9.0807) & $7.7264(4.1957-10.0277)$ & $7.5436(4.5400-10.4743)$ & $<0.001$ & $116.90 \%$ \\
\hline TNEP & $327(190-447)$ & $194(162-235)$ & $188(145-243)$ & $182(136-220)$ & $<0.001^{* *}$ & $-38.54 \% \dagger$ \\
\hline$M L$ & $0.04859(0.03868-0.06534)$ & $0.03816(0.03027-0.04640)$ & $0.03720(0.02838-0.04485)$ & $0.03701(0.02614-0.04696)$ & $<0.001$ & $-35.99 \%$ \\
\hline
\end{tabular}

SVP superficial vascular plexus, EA explant area, VA vessels area, VPA vessels percentage area, $T N J$ total number of junctions, JD junctions density, TVL total vessels length, $A V L$ average vessels length, TNEP total number of end points, $M L$ mean lacunarity. This table shows median and interquartile ranges for each parameter (25th and 75th percentile). $P$ values and percentages of change are presented one week after phacoemulsification.

Friedman ANOVA test, significant difference (bold values) was found for values with $P<0.05$

*Observed value was lower one week compared to three months after surgery

**Observed value was higher one week compared to three months after surgery 
Table 4 Statistical analysis of changes in vascular parameters in superficial vascular complex

\begin{tabular}{|c|c|c|c|c|c|c|}
\hline sVC & Before & 1 week after & 1 month after & 3 months after & $P$ & Bias \\
\hline $\mathrm{EA}\left(\mathrm{mm}^{2}\right)$ & $8.3796(8.3770-8.3806)$ & $8.3795(8.3778-8.3806)$ & $8.3801(8.3794-8.3807)$ & $8.3799(8.3787-8.3807)$ & 0.444 & $0.01 \%$ \\
\hline $\mathrm{VA}\left(\mathrm{mm}^{2}\right)$ & $3.5392(2.9247-4.4603)$ & $4.4781(3.9746-4.7150)$ & $4.5563(4.0255-4.9281)$ & $4.6398(4.2046-5.0134)$ & $<0.001$ & $22.82 \%$ \\
\hline VPA (\%) & $42.2612(34.9094-53.2560)$ & $53.4469(47.4008-56.2615)$ & $54.3727(48.0558-58.8102)$ & $55.3807(50.2028-59.8245)$ & $<0.001$ & $22.79 \%$ \\
\hline TNJ & $1025(794-1345)$ & $1320(1187-1442)$ & $1360(1215-1482)$ & $1393(1259-1501)$ & $<0.001^{*}$ & $29.51 \%^{*}$ \\
\hline JD (junctions $/ \mathrm{mm}^{2}$ ) & $122.3430(94.7414-160.8583)$ & $157.5173(141.6555-172.0434)$ & $162.3467(145.0291-176.7771)$ & $166.2187(150.3590-179.1949)$ & $<0.001^{*}$ & $29.49 \%^{*}$ \\
\hline TVL (mm) & $121.1787(104.0833-141.6036)$ & $140.6202(133.4555-146.7111)$ & $142.2354(134.4940-148.7362)$ & 144.0417 (136.0600-149.4173) & $<0.001^{*}$ & $16.71 \%^{*}$ \\
\hline $\mathrm{AVL}(\mathrm{mm})$ & $1.1413(0.6458-4.2501)$ & $4.5943(2.3966-6.4788)$ & $4.5737(2.4302-7.2035)$ & 5.5527 (3.0329-9.1357) & $<0.001^{*}$ & $166.71 \%^{*}$ \\
\hline TNEP & $477(282-621)$ & $262(212-402)$ & $234(196-379)$ & $228(190-339)$ & $<0.001^{* *}$ & $-39.56 \%{ }^{*}$ \\
\hline ML & $0.06767(0.05250-0.1128)$ & $0.04874(0.03855-0.05780)$ & $0.04805(0.03578-0.06185)$ & $0.04450(0.03148-0.05804)$ & $<0.001$ & $-44.15 \%$ \\
\hline
\end{tabular}

SVC superficial vascular complex, EA explant area, VA vessels area, VPA vessels percentage area, TNJ total number of junctions, JD junctions density, TVL total vessels length, $A V L$ average vessels length, TNEP total number of end points, $M L$ mean lacunarity

This table shows median and interquartile ranges for each parameter (25th and 75 th percentile). $P$ values and percentages of change are presented one week after phacoemulsification

Friedman ANOVA test, significant difference (bold values) was found for values with $P<0.05$

*Observed values were lower one week compared to three months after surgery

**Observed value was higher one week compared to three months after surgery

as these two layers have different hemodynamic properties.

\section{Functional hyperaemia}

Inflammatory response after uncomplicated phacoemulsification is imputed to be the cause of increased macular thickness, reaching its maximum between one week and one month after surgery and returning to baseline after 2-6 months $[2,15,16]$. However, none of these studies actually measured the local release of inflammatory metabolites. On the contrary, the prevailing view modelled from our study results' was that increase in macular hemodynamics could not be the result of postoperative inflammation as the changes persisted three months after the surgery, when inflammatory response should have been over $[2,15,17-19]$, nor decrease in IOP due to OPP consistency (Additional file 1: Table S2) [3]. Moreover, all patients underwent uncomplicated phacoemulsification with favorable, low PHACO time and
CDE (Additional file 1: Table S1), while factors contributing to neural damage and blood-brain barrier breakdown were excluded prior to enrolment.

Thus, the third mechanism, functional hyperaemia, disclosed that blood flow in retinal vasculature significantly varied due to increased intensity of light stimulus after cataract removal, in stark contrast to choroidal circulation [5]. Namely, cataract blocks up to $40 \%$ of light at different wavelengths [20]. Increase in metabolism which accompanies neuronal activity lowers $\mathrm{O}_{2}$ and glucose levels and leads to production of vasoactive metabolites [21]. Products of neuronal activity adenosine, lactate and arachnoidic acid cause vasodilatation and functional hyperaemia to compensate for energy consumption and increase in ganglion cell activity due to light stimulation restoring $\mathrm{O}_{2}$ and glucose levels $[5,22$, 23]. Likewise, larger blood flow is induced after a period of dark adaptation [23], what in our case, hypothetically, we could consider cataract to be as cataracts gradually weaken the intensity of light stimuli.

Table 5 Statistical analysis of changes in vascular parameters in intermediate capillary plexus

\begin{tabular}{|c|c|c|c|c|c|c|}
\hline ICP & Before & 1 week after & 1 month after & 3 months after & $P$ & Bias \\
\hline $\mathrm{EA}\left(\mathrm{mm}^{2}\right)$ & 8.3800 (8.3774-8.3806) & 8.3797 (8.3779-8.3808) & 8.3801 (8.3788-8.3809) & 8.3800 (8.3786-8.3808) & 0.359 & $0.01 \%$ \\
\hline$V A\left(m^{2}\right)$ & 4.4108 (3.7545-4.8631) & $4.8974(4.6741-5.1877)$ & $4.9264(4.5317-5.1928)$ & $5.0351(4.7020-5.3053)$ & $<0.001$ & $16.06 \%$ \\
\hline VPA (\%) & $52.6315(44.8284-58.0264)$ & 58.4357 (55.8038-62.1985) & $58.7958(54.0681-61.9713)$ & $60.0813(56.1073-63.3031)$ & $<0.001$ & $16.02 \%$ \\
\hline TNJ & $1446(1197-1576)$ & $1593(1504-1675)$ & $1603(1452-1682)$ & $1619(1506-1670)$ & $<0.001$ & $16.46 \%$ \\
\hline JD (junctions/mm²) & $172.6665(142.9233-188.0191)$ & 190.1043 (179.5759-200.7896) & $191.3712(173.2411-200.7275)$ & 193.1983 (179.7861-202.8178) & $<0.001$ & $16.42 \%$ \\
\hline TVL (mm) & $145.8206(129.0303-153.5934)$ & $154.3064(150.4366-158.9588)$ & $155.3216(146.2016-158.3196)$ & 155.9789 (148.7249-160.2269) & $<0.001$ & $10.80 \%$ \\
\hline $\mathrm{AVL}(\mathrm{mm})$ & $3.1665(1.1356-5.2864)$ & $6.2908(4.1408-8.3364)$ & $6.2008(3.8223-8.0361)$ & 6.2737 (3.8795-8.4574) & $<0.001$ & $75.78 \%$ \\
\hline TNEP & $366(236-550)$ & $233(191-296)$ & $236(202-313)$ & $231(190-304)$ & $<0.001$ & $-36.58 \%$ \\
\hline MEL & $0.03303(0.02445-0.05412)$ & $0.02367(0.02006-0.02980)$ & $0.02469(0.01956-0.03311)$ & $0.02373(0.01769-0.03048)$ & $<0.001$ & $-50.61 \%$ \\
\hline
\end{tabular}

ICP intermediate capillary plexus, EA explant area, VA vessels area, VPA vessels percentage area, $T N J$ total number of junctions, JD junctions density, TVL total vessels length, $A V L$ average vessels length, TNEP total number of end points, $M L$ mean lacunarity

This table shows median and interquartile ranges for each parameter (25th and 75th percentile). $P$ values and percentages of change are presented one week after phacoemulsification

Friedman ANOVA test, significant difference (bold values) was found for values with $P<0.05$ 
Table 6 Statistical analysis of changes in vascular parameters in deep vascular complex

\begin{tabular}{|c|c|c|c|c|c|c|}
\hline DVC & Before & 1 week after & 1 month after & 3 months after & $P$ & Bias \\
\hline $\mathrm{EA}\left(\mathrm{mm}^{2}\right)$ & $8.3798(8.3767-8.3807)$ & $8.3799(8.3788-8.3808)$ & $8.3804(8.3791-8.3810)$ & 8.3799 (8.3786-8.3808) & 0.128 & $0.02 \%$ \\
\hline $\mathrm{VA}\left(\mathrm{mm}^{2}\right)$ & $4.6295(3.7813-5.1470)$ & $5.2785(5.0204-5.5748)$ & $5.3497(4.9653-5.6558)$ & $5.3920(4.9867-5.7371)$ & $<0.001$ & $18.17 \%$ \\
\hline VPA (\%) & $55.2483(45.1905-61.4192)$ & 63.0098 (59.9148-66.5252) & $63.8886(59.2817-67.4735)$ & $64.3579(59.5031-68.4626)$ & $<0.001$ & $18.10 \%$ \\
\hline TNJ & $1601(1270-1665)$ & $1716(1670-1771)$ & $1729(1647-1772)$ & $1722(1634-1780)$ & $<0.001$ & $15.41 \%$ \\
\hline JD (junctions $/ \mathrm{mm}^{2}$ ) & 191.0277 (151.6214-198.5983) & 204.7507 (199.3202-211.4425) & 206.7926 (196.6997-211.4956) & $205.4722(194.6548-212.3658)$ & $<0.001$ & $15.34 \%$ \\
\hline TVL (mm) & 152.8660 (130.5779-158.5100) & 160.3862 (156.7585-163.6309) & 161.0969 (156.1049-163.2658) & $160.5890(155.0713-163.9713)$ & $<0.001$ & $10.74 \%$ \\
\hline $\mathrm{AVL}(\mathrm{mm})$ & $3.8988(0.9151-10.1915)$ & $11.0566(6.7885-23.2487)$ & $13.4610(7.3215-20.4125)$ & $12.1796(7.0645-20.5614)$ & $<0.001$ & $113.40 \%$ \\
\hline TNEP & $333(177-525)$ & $163(117-221)$ & $155(115-225)$ & $140(112-213)$ & $<0.001$ & $-49.04 \%$ \\
\hline ML & $0.03602(0.02654-0.07429)$ & $0.02452(0.01976-0.03203)$ & $0.02530(0.01902-0.03206)$ & $0.02564(0.01882-0.03265)$ & $<0.001$ & $-53.41 \%$ \\
\hline
\end{tabular}

DVC deep vascular complex, EA explant area, VA vessels area, VPA vessels percentage area, TNJ total number of junctions, JD junctions density, TVL total vessels length, $A V L$ average vessels length, TNEP total number of end points, $M L$ mean lacunarity

This table shows median and interquartile ranges for each parameter (25th and 75th percentile). $P$ values and percentages of change are presented one week after phacoemulsification

Friedman ANOVA test, significant difference (bold values) was found for values with $P<0.05$

\section{SVC vs DVC}

The greatest increase in VA, VPA, TNJ, JD, TVL and AVL was found in SVC (Additional file 1: Table S4). Due to functional hyperaemia, AVL increased more than TVL suggesting coiling of blood vessels (Additional file 1: Table S4).

On the contrary, the greatest change of $\mathrm{ML}$ as an index for vascular structural non-uniformity, [12] appeared in DVC (Additional file 1: Table S4). These differences suggest different metabolic demands of retinal layers supplied by SVC and DVC.

NFLVP, a layer of long capillaries with small number of anastomosis located only in the posterior pole [6,24], is presented with unique configuration (Additional file 1: Table S7) manifesting the highest number of end points and the lowest number of junctions (Table 2; Additional file 1: Table S7; Table S9).

Three months after surgery VA, VPA, TNJ, JD, TVL and AVL were significantly higher in DVC compared to SVC, while TNEP and ML were significantly higher in SVC (Additional file 1: Table S10). On the contrary, while comparing SVP and DCP (Additional file 1: Table S8), greater VA and VPA for SVP network were found likewise [10], but DCP showed higher TNJ, JD and TVL. Aforesaid attributes could correlate to the larger diameter of the SVP vasculature opposed to DVP being solely capillary meshwork [25]. Morphometry of ICP and DCP did not demonstrate significant differences (Additional file 1: Table S8).

Until this date, only two OCT-A studies analysed retinal blood vessels after uncomplicated phacoemulsification $[2,3]$. Performed on a relatively small subject samples $(N=9$ and $N=32)$, they used different OCT-A devices while $\mathrm{Q}$ and EA comparison before and after cataract surgery was not performed [2, 3]. Zhao et al. observed parafoveal and perifoveal blood vessels density increase one week after phacoemulsification lasting up to three months after surgery [3]. Furthermore, cataract surgery was followed by a decrease in the foveal avascular zone surface, and an increase in full and inner retinal thickness, occurring one week after surgery, and still increasing up to three months after the surgery [3]. Outer

Table 7 Statistical analysis of changes in vascular parameters in deep capillary plexus

\begin{tabular}{|c|c|c|c|c|c|c|}
\hline DCP & Before & 1 week after & 1 month after & 3 months after & $P$ & Bias \\
\hline$E A\left(\mathrm{~mm}^{2}\right)$ & 8.3792 (8.3766-8.3803) & 8.3802 (8.3790-8.3808) & 8.3802 (8.3789-8.3807) & 8.3801 (8.3790-8.3810) & 0.357 & $0.01 \%$ \\
\hline$V A\left(\mathrm{~mm}^{2}\right)$ & 4.2319 (3.4769-4.8241) & 4.9539 (4.4996-5.2425) & $4.9380(4.5294-5.3485)$ & $4.9121(4.5870-5.4160)$ & $<0.001^{*}$ & $17.65 \%{ }^{*}$ \\
\hline VPA (\%) & 50.5055 (41.6312-57.5592) & 59.1286 (53.7127-62.5724) & $58.9327(54.1117-63.8239)$ & $58.6169(54.7285-64.6397)$ & $<0.001^{*}$ & $17.59 \%{ }^{*}$ \\
\hline TNJ & $1375(1078-1546)$ & $1573(1442-1646)$ & $1572(1446-1650)$ & $1572(1440-1654)$ & $<0.001$ & $16.77 \%$ \\
\hline JD (junctions/mm²) & 164.1052 (128.8104-184.4084) & $187.7513(172.1331-196.4872)$ & 187.7643 (172.532-196.884) & $187.5586(171.8801-197.3193)$ & $<0.001$ & $16.71 \%$ \\
\hline TVL (mm) & $138.1963(120.0253-150.4007)$ & $151.3618(144.6869-156.4053)$ & $152.0403(145.4315-156.7864)$ & $151.0493(145.3439-156.7136)$ & $<0.001$ & $11.00 \%$ \\
\hline $\mathrm{AVL}(\mathrm{mm})$ & $1.9423(0.7977-4.6726)$ & $5.8517(3.7833-8.4856)$ & $5.3625(3.2110-8.1661)$ & $5.2044(3.3965-8.1440)$ & $<0.001$ & $103.51 \%$ \\
\hline TNEP & $411(237-597)$ & $239(180-328)$ & $219(171-311)$ & $225(167-298)$ & $<0.001$ & $-37.06 \%$ \\
\hline$M L$ & $0.05597(0.04235-0.08264)$ & $0.04124(0.0304-0.05118)$ & $0.03980(0.03073-0.04955)$ & 0.03709 (0.03059-0.05586) & $<0.001$ & $-44.00 \%$ \\
\hline
\end{tabular}

$D C P$ deep capillary plexus, EA explant area, $V A$ vessels area, $V P A$ vessels percentage area, $T N J$ total number of junctions, $J D$ junctions density, $T V L$ total vessels length, $A V L$ average vessels length, TNEP total number of end points, $M L$ mean lacunarity

This table shows median and interquartile ranges for each parameter ( 25 th and 75 th percentile). $P$ values and percentages of change are presented one week after phacoemulsification

Friedman ANOVA test, significant difference (bold values) was found for values with $P<0.05$

*Observed values were lower one week than three months after surgery 


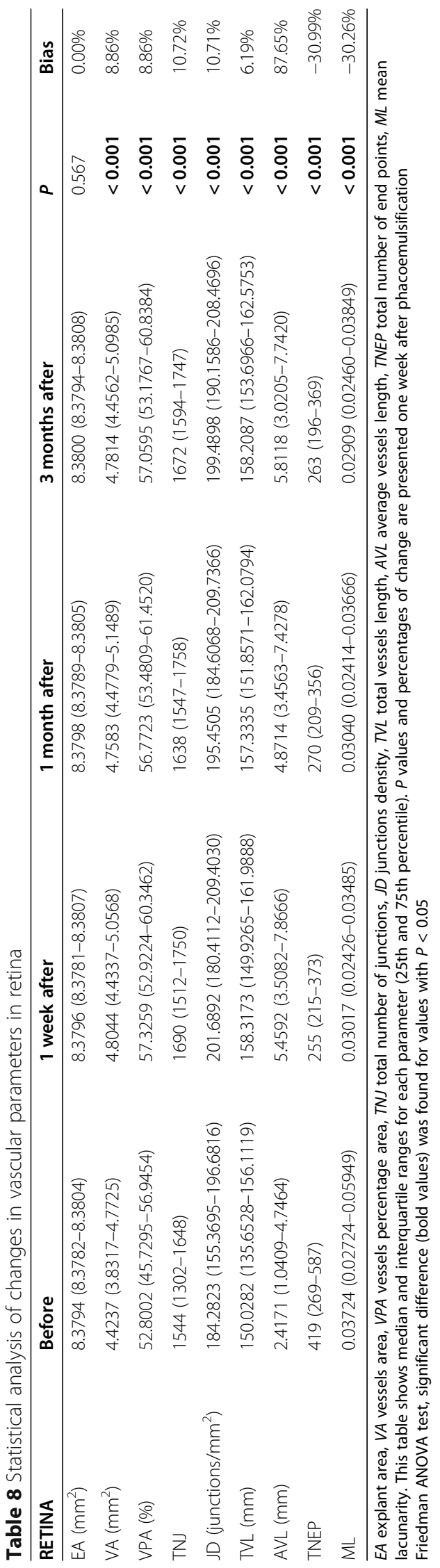


retinal thickness remained almost unchanged [3]. The vascular pattern responses observed here could give the anatomical background for the aforementioned results. Although all retinal layers demonstrated increase in perfusion, SVC underwent greater change than DVC (Additional file 1: Table S4). Inner plexiform layer contains both superficial and deep blood vessels [6]. Thus, the functional hyperaemia observed could cause an increase in full and inner retinal thickness [3]. On the contrary, we hypothesize that functional hyperaemia identified in DCP cannot elicit significant changes of outer retinal thickness as DCP is intersected between INL and OPL [6], justifying Zhao's et al. results [3].

\section{Choroid}

CC presented with the greatest VA, VPA, TNJ, JD, TVL and AVL, and the lowest TNEP and ML (Additional file 1: Table S5; Table S9) confirming its structure as a continuous capillary meshwork with a high number of anastomosis [25]. Opposed to the retina, we found no significant changes in the choroid (Additional file 1: Table S6) and CC (Additional file 1: Table S5), except for TNEP and ML in CC. Light stimulation has a little effect on choroidal circulation, insensitive to $\mathrm{pO}_{2}$ fluctuations [5]. We thus concluded that physiological requirements outlined with low PHACO time and CDE did not reach the threshold to induce outer blood-retinal barrier breakdown and inflammatory response. Further, significant decrease of TNEP and ML in CC (Additional file 1: Table S5) could result from increased demand in heat dissipation through opening of anastomoses corroborating with published studies $[2,16,26]$. Some authors hypothesized increase in subfoveal thickness to be a consequence of local choroidal inflammatory response but found no reasoning for these changes, while correlation to CDE was not reported [18, 25, 27].

\section{Ageing}

Ageing causes altered hemodynamics and hypoperfusion mostly in neural tissues with high metabolic demand [28]. Decline in metabolic activity under physiological ageing was further supported by our study correspondingly, as retinal macular perfusion significantly increased after uncomplicated phacoemulsification.

\section{Image quality}

One might ask if these changes in macular perfusion after surgery were the result of better image quality after cataract removal. Thus, we counterbalanced this potential bias. OCT-A may overcome early stage cataract in contrast to clinically significant ones [29]. As follows, this study included only patients with mild to moderate opacities, graded objectively (Table S1). Secondly and more important, OCT-A image quality (Q) quantified by the software integrated in HRA + OCT Spectralis ${ }^{\circ}$ before and after surgery was statistically the same (Table 1). Consequently, perfusion changes demonstrated here were unlikely the result of the improvement of the ocular optics after cataract removal. Furthermore, different time frames of perfusion alterations were demonstrated (Tables 2-8; Additional file 1: Table S5). In addition, the latest software version of Spectralis ${ }^{\circ}$ uses the Position Artefact Removal tool, eliminating blood movement artefacts and enabling even more distinct analysis of deeper layers [9]. With TruTrack Active Eye Tracking technology high-quality retinal imaging even with eye movements is allowed [9].

There are few limitations to this analysis. First, we reported the values for only 55 subjects of Caucasian descent for whom we did not measure retinal metabolic activity. Further studies using OCT-A are needed to establish a normative database of observed vascular parameters for other demographic variables. Second, the analysis of retinal and choroidal pathology was beyond the scope of this report.

\section{Conclusions}

This is the first OCT-A study that has clearly demonstrated persistent increase in macular perfusion most likely due to functional hyperaemia. We presume the effect is evoked by increased light intensity stimulation of retina after cataract removal. In this study, phacoemulsification in elderly population proved as an advantageous feature in addition to restoring visual acuity. This beneficial event could facilitate the decision-making process with regard to earlier timing for cataract removal in healthy aging patients. Further studies with a longer follow-up period are warranted to validate our results and reveal temporal trends. Thus, to conclude if functional hyperaemia is a long-term condition.

\section{Supplementary Information}

The online version contains supplementary material available at https://doi. org/10.1186/s12886-021-01837-2.

\footnotetext{
Additional file 1: Table S1 General characteristics of patients and surgery parameters. Table S2 Pressure parameters and visual acuity changes. Table $\mathbf{S 3}$ Determined changes in vascular parameters in corresponding layers. Table S4 Percentage of determined changes in vascular parameters in corresponding layers. Table S5 Statistical analysis of changes in vascular parameters in choriocapillaris. Table S6 Statistical analysis of changes in vascular parameters in choroid. Table S7 Morphometric differences between nerve fiber layer and superficial vascular plexus, intermediate capillary plexus and deep capillary plexus three months after surgery. Table S8 Morphometric differences between superficial vascular plexus, intermediate capillary plexus and deep capillary plexus three months after surgery. Table S9 Morphometric differences between choriocapillaris and nerve fiber layer vascular plexus, superficial vascular plexus, intermediate capillary plexus and deep capillary plexus three months after surgery. Table S10 Morphometric differences between deep and superficial vascular complex three months
} 
after surgery. Table S11 OCT-A vascular parameters before and one week after phacoemulsification.

\section{Abbreviations}

$A L$ : Axial length; $A V L$ : Average vessels length; BCVA: Best corrected visual acuity; CC: Choriocapillaris; CDE: Cumulative dissipated energy; DBP: Diastolic blood pressure; DCP: Deep capillary plexus; DVC: Deep vascular complex; EA: Explant area; GCL: Ganglion cell layer; ICP: Intermediate capillary plexus; INL: Inner nuclear; IOP: Intraocular pressure; IPL: Inner plexiform layer; JD: Junctions density; MAP: Mean arterial pressure; ML: Mean lacunarity; NFL: Nerve fiber layer; NFLVP: Nerve fiber layer vascular plexus; OCT: Optical coherence tomography; OCT-A: Optical coherence tomography angiography; OPL: Outer plexiform layer; OPP: Ocular perfusion pressure; PHACO time: Total ultrasound time; PME: Pseudophakic macular oedema; PNS: Pentacam ${ }^{\oplus}$ Nucleus Staging; Q: Images quality index; SBP: Systolic blood pressure; SVC: Superficial vascular complex; SVP: Superficial vascular plexus; TNEP: Total number of end points; TNJ: Total number of junctions; TVL: Total vessels length; VA: Vessels area; VPA: Vessels percentage area

\section{Acknowledgements}

Not applicable.

\section{Authors' contributions}

Conception and design of the study: AK, MBj, MB, NV. Analysis and interpretation: $A K, M B j, M B, B K E, B R, N V$. Writing of the article: $A K, M B j ;$ Critical revision of the article: $A K, M B j, M B, B K E, B R, N V$. Data collection: AK, BKE. Provision of patients. AK. Statistical expertise: $A K, M B j, B R$. Literature search: $A K, M B j, M B, B K E, B R$. Administrative support: AK, MB, BKE, BR. All authors read and approved the final manuscript.

\section{Funding}

This research did not receive any funding.

\section{Availability of data and materials}

The datasets generated and/or analysed during the current study are not publicly available due to extensive and large-scale datasheets but are available from the corresponding author on reasonable request.

\section{Ethics approval and consent to participate}

The study protocol was approved by the Institutional Review Board of University Hospital "Sveti Duh", Zagreb, Croatia (IRB no. 01-4212/2) and was conducted in accordance with the tenets of the World Medical Association Declaration of Helsinki. Written informed consent to participate was obtained from all patients before inclusion in the study.

\section{Consent for publication}

Not applicable.

\section{Competing interests}

The authors declare that they have no competing interests.

\section{Author details}

${ }^{1}$ Department of Ophthalmology, Reference Centre of the Ministry of Health of the Republic of Croatia for Paediatric Ophthalmology and Strabismus, University Hospital "Sveti Duh", Sveti Duh 64, Zagreb, Croatia. 'Faculty of Dental Medicine and Health Osijek, Josip Juraj Strossmayer University of Osijek, Osijek, Croatia. ${ }^{3}$ Faculty of Medicine, Josip Juraj Strossmayer University of Osijek, Osijek, Croatia. ${ }^{4}$ University of Zagreb School of Medicine, Zagreb, Croatia. ${ }^{5}$ Department of Ophthalmology, University Hospital Centre Zagreb, Zagreb, Croatia.

Received: 28 November 2020 Accepted: 18 January 2021 Published online: 05 February 2021

\section{References}

1. Micieli JA, Arshinoff SA. Cataract surgery. CMAJ. 2011;183:1621.

2. Pilotto E, Leonardi F, Stefanon G, Longhin E, Torresin T, Deganello D, et al. Early retinal and choroidal OCT and OCT angiography signs of inflammation after uncomplicated cataract surgery. Br J Ophthalmol. 2019;103:1001-7.
3. Zhao Z, Wen W, Jiang C, Lu Y. Changes in macular vasculature after uncomplicated phacoemulsification surgery: optical coherence tomography angiography study. J Cataract Refract Surg. 2018;44:453-8.

4. Rich WJ, James ML. Cataract surgery and vascular changes in the eye. Acta Ophthalmol Suppl. 1989;191:39-42.

5. Kur J, Newman EA, Chan-Ling T. Cellular and physiological mechanisms underlying blood flow regulation in the retina and choroid in health and disease. Prog Retin Eye. 2012;31:377-406.

6. Coscas F, Sellam A, Glacet-Bernard A, Jung C, Goudot M, Miere A, et al. Normative data for vascular density in superficial and deep capillary plexuses of healthy adults assessed by optical coherence tomography angiography. Invest Ophthalmol Vis Sci. 2016;57:211-23.

7. Harjasouliha A, Raiji V, Garcia Gonzalez JM. Review of hypertensive retinopathy. Dis Mon. 2017;63:63-9.

8. Kniestedt C, Stamper RL. Visual acuity and its measurement. Ophthalmol Clin N Am. 2003;16:155-70.

9. Spectralis Product Family. User Manual Softvare version 6.8. Heidelberg: Heidelberg Engineering GmbH; 2017.

10. Hosari S, Hohberger B, Theelke L, Sari H, Lucio M, Mardin CY. OCT angiography: measurement of retinal macular microvasculature with Spectralis II OCT angiography - reliability and reproducibility. Ophthalmologica. 2020;243:75-84.

11. Lupidi M, Coscas F, Cagini C, Fiore T, Spaccini E, Fruttini D, et al. Automated quantitative analysis of retinal microvasculature in Normal eyes on optical coherence tomography angiography. Am J Ophthalmol. 2016;169:9-23.

12. Zudaire E, Gambardella L, Kurcz C, Vermeren S. A computational tool for quantitative analysis of vascular networks. PLoS One. 2011;6:e27385.

13. Oh LJ, Nguyen C, Wong E, Wang SSY. Prospective study of centurion ${ }^{\odot}$ versus Infiniti ${ }^{\circledast}$ phacoemulsification systems: surgical and visual outcomes. Int J Ophthalmol. 2017;10:1698-702.

14. Kim SJ, Belair ML, Bressler NM, Dunn JP, Thorne JE, Kedhar SR, et al. A method of reporting macular edema after cataract surgery using optical coherence tomography. Retina. 2008;28:870-6.

15. Gharbiya M, Cruciani F, Cuozzo G, Parisi F, Russo P, Abdolrahimzadeh S. Macular thickness changes evaluated with spectral domain optical coherence tomography after uncomplicated phacoemulsification. Eye (Lond). 2013;27:605-11.

16. Falcão MS, Gonçalves NM, Freitas-Costa P, Beato JB, Rocha-Sousa A, Carneiro A, et al. Choroidal and macular thickness changes induced by cataract surgery. Clin Ophthalmol. 2014;8:55-60.

17. Neatrour K, McAlpine A, Owens TB, Trivedi RH, Poole Perry L. Evaluation of the etiology of persistent iritis after cataract surgery. Ophthalmic Inflamm Infect. 2019;9:4

18. Ibrahim AM, Elgouhary SM, Nassar MK, El Batanony AH. Changes in Choroidal thickness after cataract surgery. Semin Ophthalmol. 2018;33: 664-70.

19. Alio JL, Bodaghi B, Tassignon MJ. Guidelines for managing post-cataract surgery inflammation: can we reach a consensus? Ophthalmology times Europe. 2008. https://www.ophthalmologytimes.com/sites/default/files/lega cy/mm/OphthalmologyTimesEurope/PDF/Guidelines-for-managing-post-ca taract-surgery-inflammation.pdf. Accessed 25 Sep 2020.

20. Artigas JM, Felipe A, Navea A, Fandiño A, Artigas C. Spectral transmission of the human crystalline lens in adult and elderly persons: color and total transmission of visible light. Invest Ophthalmol Vis Sci. 2012;53:4076-84.

21. Roy CS, Sherrington CS. On the regulation of the blood-supply of the brain. J Physiol. 1890;11:85-158.

22. Attwell D, Buchan A, Charpak S, Lauritzen M, Macvicar BA, Newman EA. Glial and neuronal control of brain blood flow. Nature. 2010;468:232-43.

23. Noonan JE, Lamoureux EL, Sarossy M. Neuronal activity-dependent regulation of retinal blood flow. Clin Exp Ophthalmol. 2015;43:673-82.

24. Chan G, Balaratnasingam C, Yu PK, Morgan WH, McAllister IL, Cringle SJ, et al. Quantitative morphometry of perifoveal capillary networks in the human retina. Invest Ophthalmol Vis Sci. 2012;53:5502-14.

25. Zeng S, Liang C, He Y, Zhao Q, Dai S, Cheng F, et al. Changes of Subfoveal Choroidal thickness after cataract surgery: a meta-analysis. J Ophthalmol. 2018;2018:2501325.

26. Brito PN, Rosas VM, Coentrão LM, Carneiro ÂV, Rocha-Sousa A, Brandão E, et al. Evaluation of visual acuity, macular status, and subfoveal choroidal thickness changes after cataract surgery in eyes with diabetic retinopathy. Retina. 2015;35(2):294-302. 
27. Chen H, Wu Z, Chen Y, He M, Wang J. Short-term changes of choroidal vascular structures after phacoemulsification surgery. BMC Ophthalmol. 2018;18:81.

28. Lin Y, Jiang H, Liu Y, Rosa Gameiro G, Gregori G, Dong C, et al. Age-related alterations in retinal tissue perfusion and volumetric vessel density. Invest Ophthalmol Vis Sci. 2019:60:685-93.

29. Yu S, Frueh BE, Steinmair D, Ebneter A, Wolf S, Zinkernagel MS, et al. Cataract significantly influences quantitative measurements on sweptsource optical coherence tomography angiography imaging. PLoS One. 2018;13(10):e0204501.

\section{Publisher's Note}

Springer Nature remains neutral with regard to jurisdictional claims in published maps and institutional affiliations.

Ready to submit your research? Choose BMC and benefit from:

- fast, convenient online submission

- thorough peer review by experienced researchers in your field

- rapid publication on acceptance

- support for research data, including large and complex data types

- gold Open Access which fosters wider collaboration and increased citations

- maximum visibility for your research: over $100 \mathrm{M}$ website views per year

At BMC, research is always in progress.

Learn more biomedcentral.com/submissions 